\title{
A novel loop-mediated isothermal amplification method for efficient and robust detection of EGFR mutations
}

\author{
SHO HORIUCHI $^{1,2}$, YUICHI SAITO ${ }^{2,3}$, ATSUKA MATSUI ${ }^{4}$, NOBUMASA TAKAHASHI ${ }^{3}$, \\ TOMOHIKO IKEYA $^{3}$, EISHIN HOSHI ${ }^{3}$, YOSHIHIKO SHIMIZU ${ }^{5}$ and MASANORI YASUDA ${ }^{1}$ \\ ${ }^{1}$ Department of Pathology, Saitama Medical University International Medical Center, Hidaka 350-1298; \\ ${ }^{2}$ Department of Thoracic Surgery, Saitama Cardiovascular and Respiratory Center, Kumagaya, Saitama 360-0197; \\ ${ }^{3}$ Department of Surgery, Teikyo University School of Medicine, Tokyo 173-8605; ${ }^{4}$ Fundamental Research Laboratory, \\ Fundamental Technology Research Department, Eiken Chemical Co., Ltd., Otawara, Tochigi 324-0036; \\ ${ }^{5}$ Department of Pathology, Saitama Cardiovascular and Respiratory Center, Kumagaya, Saitama 360-0197, Japan
}

Received July 18,2019; Accepted December 12, 2019

DOI: 10.3892/ijo.2020.4961

\begin{abstract}
The activation of somatic mutations conferring sensitivity to epidermal growth factor receptor (EGFR) tyrosine kinase inhibitors has been widely used in the development of advanced or metastatic primary lung cancer therapy. Therefore, identification of EGFR mutations is essential. In the present study, a loop-mediated isothermal amplification (LAMP) method was used to identify EGFR mutations, and its efficiency was compared with the Therascreen quantitative PCR assay. Using LAMP and Therascreen to analyze surgically resected tissue samples from patients with pulmonary adenocarcinoma, EGFR mutations were observed in 32/59 tumor samples (LAMP) and 33/59 tumor samples (Therascreen). Notably, the LAMP assay identified one tumor as wild-type, which had previously been identified as a deletion mutation in exon 19 via the Therascreen assay (Case X). However, the direct sequencing to confirm the EGFR status of the Case $\mathrm{X}$ adhered to the results of the LAMP assay. Further experiments using Case X DNA identified this exon 19 deletion mutation using both methods. In addition, a novel deletion mutation in exon 19 of the EGFR was identified. Overall, the present study shows that the LAMP method may serve as a valuable alternative for the identification oncogene mutations.
\end{abstract}

Correspondence to: Dr Yuichi Saito, Department of Thoracic Surgery, Saitama Cardiovascular and Respiratory Center, 1696 Itai, Kumagaya, Saitama 360-0197, Japan

E-mail:k3699004@gmail.com

Abbreviations: EGFR, epidermal growth factor receptor; FFPE, formalin-fixed, paraffin-embedded; NSCLC, non-small cell lung cancer; LAMP, loop-mediated isothermal amplification; PPV, positive predictive value; NPV, negative predictive value; PCR, polymerase chain reaction; TKI, tyrosine kinase inhibitors

Key words: EGFR, LAMP, NSCLC, adenocarcinoma, PCR

\section{Introduction}

Lung cancer has the highest morbidity and mortality of all malignancies in the USA in 2011 and 2013 (1,2). Non-small cell lung carcinoma (NSCLC) is the most common form of lung cancer, accounting for $70-85 \%$ of cases and is primarily treated using systemic chemotherapy (3). However, NSCLC treatment has evolved due to the development of therapy targeting the activation of mutations in the epidermal growth factor receptor (EGFR) (4).

EGFR activation is caused by genetic mutations, conferring susceptibility to EGFR tyrosine kinase inhibitor (TKI) treatment, and was first reported in 2004 (5). EGFR mutations of pulmonary adenocarcinoma are present in $5-15 \%$ of the Caucasian population and $40-55 \%$ of the East Asian population (6-8). Clinical trials have shown that patients with pulmonary adenocarcinoma with an EGFR mutation exhibit clinical responses to orally administered EGFR inhibitors $(5,7,9)$. In 2016, Lin et al (4) reported that 20/137 (14.6\%) patients with EGFR-mutant metastatic lung adenocarcinoma had a survival time of 5-years (4). Therefore, detection of EGFR mutations is an important step in the treatment-decision pathway for patients with pulmonary adenocarcinoma.

Previously, direct DNA sequencing was the standard method for detecting genetic mutations (10); however, at present, several alternative methods for mutation testing have been developed $(11,12)$. For example, the Therascreen EGFR PCR kit ${ }^{\circledR}$ (Qiagen, Inc.) is a commercial quantitative (q)PCR kit and has been widely adopted for clinical practice; however, this method is time-consuming and possesses certain procedural complexities, for example, requiring several temperature changes during DNA amplification (11). Next-generation sequencing has improved the efficiency of oncogene testing by high-throughput sequencing, which can detect dozen of mutations at the same time, but the high-cost of this technique limits its clinical usage $(13,14)$. Therefore, detecting oncogenic mutations using a simple, easy and highly reproducible method remains a challenge.

Loop-mediated isothermal amplification (LAMP) is a new PCR based method with high levels of specificity and 
amplification efficiency and utilizes six primers (15). This method is performed under isothermal conditions, thereby enabling rapid amplification. Due to the high specificity and rapid detection quality of LAMP, this method has been widely used in the fields of bacteriology (16) and virology $(17,18)$. However, to the best of our knowledge, there are very few studies reporting the value of LAMP in determining EGFR mutations. Therefore, the present study aimed to detect EGFR mutations in surgically resected tumor tissues from patients with pulmonary adenocarcinoma using this method, as detection of EGFR mutations is one a key examination for patients with pulmonary adenocarcinoma $(4,5,7,9)$. In addition, the sensitivity, specificity, positive predictive value, negative predictive value and accuracy of LAMP was evaluated and compared with the Therascreen EGFR PCR kit ${ }^{\circledR}$.

\section{Materials and methods}

Tumor tissue samples. The tumor tissues were surgically resected from 189 consecutive patients diagnosed with pulmonary adenocarcinoma by the expert pathologist at The Saitama Cardiovascular and Respiratory Center (Kumagaya, Japan) between January 2016 and October 2017. All pathological diagnosis was determined on the basis of the WHO classification version 8 (19) by an expert pathologist, who normally makes a diagnosis using HE-stained slides using light microscope (Nikon Co., ECLIPSE Ni-u) from a low magnification to a high magnification. The inclusion criteria were as follows: i) Surgically resected tissue of primary lung cancer; ii) pulmonary adenocarcinoma; iii) enough volume materials for molecular testing; and iv) informed written consent from patients. Conversely, cases with no informed consent or less volume sample were excluded. Clinical characteristics of the 59 patients are presented in Table I. The mean patient age was 69.6 years and included 28 males and 31 females. All samples were fixed with $10 \%$ buffer formalin at room temperature (24-36 h) to create formalin-fixed, paraffin-embedded (FFPE) tumor blocks at Department of Pathology in Saitama Cardiovascular and Respiratory Center. Hematoxylin-eosin staining was performed by the standard method using Tissue-Tek Prisma (Sakura Finetek Japan Co., Ltd.) according to the manufacturer's protocol. Prior to DNA extraction, the tumor content of each sample was assessed using light microscopy at $\times 10$ and $\times 100$ magnification, to ensure efficient PCR amplification. After sections were deparaffinized with xylene and hydrated through a graded series of ethanol $(100,100,85$ and $70 \%$ ethanol), DNA from the tissue blocks was extracted using the QIAampTM DNA FFPE Tissue kit ${ }^{\circledR}$ (Qiagen, Inc.) and analyzed using a QIAcube Robot ${ }^{\circledR}$ (Qiagen, Inc.) according to the manufacturer's protocols (20).

Therascreen qPCR mutation analysis. The presence of EGFR mutations was determined using a Therascreen EGFR PCR kit ${ }^{\mathrm{B}}$ (Qiagen, Inc.) according to the manufacturer's protocols (21).

\section{LAMP mutation analysis}

Primer design. A primer set for LAMP amplification of the partial sequence of the EGFR gene (NG_007726) was designed using Primer Explorer (primerexplorer.jp/e/). Fig. 1 presents the sequences of the primers used with forward and backward outer primers (F3 and B3), forward and backward inner primers (FIP and BIP) and forward and back-loop primers (LF and LB). The primers were synthesized and purified by Eurofin Genomics. Block oligo and fluorophore-labelled probes were synthesized and purified by Japan Bio Services Co., Ltd., or Gene Design, Inc.

LAMP assay. LAMP assays were performed using the 25 reaction volume with $30 \mathrm{mM} \mathrm{KCl}, 1.8 \%$ Dextran, $14 \mathrm{mM}$ Tricine- $\mathrm{NaOH}$ (pH 8.8), 0.1\% n-heptyl, 0.5\% Tween-20, 0.3\% Fish collagen peptide, $1 \mathrm{mM}$ DTT, $1.7 \mathrm{mM}$ each dNTPs, $8.2 \mathrm{mM}$ $\mathrm{MgSO} 4,1.6 \mu \mathrm{M}$ each of the forward and backward inner primer, $0.8 \mu \mathrm{M}$ each of the forward and backward loop primer, $0.2 \mu \mathrm{M}$ forward and backward outer primer, $1.2 \mu \mathrm{M}$ block oligo, $0.04 \mu \mathrm{M}$ fluorophore-labelled probe, $0.0375 \mathrm{x}$ Gel green and 12.3 units Bst DNA polymerase. $\mathrm{KCl}$, Dextran, Trincine $\mathrm{NaOH}, \mathrm{n}$-heptyl, Tween-20, Fish collagen peptide, DTT, and $\mathrm{MgSO} 4$ were obtained from FUJIFILM Wako Pure Chemical Corporation (http://ffwk.fujifilm.co.jp/en/index.html). dNTPs were obtained from NIPPON GENE CO., Ltd. (https://www. nippongene.com/english/index.html). Gel green was obtained from Biotium (https://biotium.com/). Bst DNA polymerase was obtained from New England Biolabs Inc. (https://international. neb.com). The LAMP reaction was run for $120 \mathrm{~min}$ at $65^{\circ} \mathrm{C}$ using a LightCycler $480^{\circledast}$ (Roche Diagnostics K.K.).

Detection of the LAMP products. Melting curve analysis was used to detect LAMP products. The LAMP amplicons were denatured at $95^{\circ} \mathrm{C}$ for $5 \mathrm{~min}$, followed by hybridization at $37^{\circ} \mathrm{C}$ for $5 \mathrm{~min}$. Subsequently, the temperature was gradually raised to $80^{\circ} \mathrm{C}$ and the fluorescent intensity was measured 7 times per $1{ }^{\circ} \mathrm{C}$ increment. The obtained data were analyzed by the LightCycler $480^{\circledR}$ software (version 1.5.1.62; https://lifescience.roche.com/global_en/products/lightcycler14301-480-software-version-15.html) to calculate melting peak.

Sensitivity of LAMP and Therascreen assays. In order to evaluate the analytical sensitivity, an artificial gene harboring an EGFR L858R mutation (c.2573T >G) as a plasmid DNA was obtained from Eurofin Genomics. The plasmid DNA that had been linearized using EcoR V and Xho I were extracted and purified from $1 \%$ agarose gel after 40 min electrophoresis with $100 \mathrm{~V}$. The agarose gel was stained by $0.5 \mu \mathrm{g} / \mathrm{ml}$ ethidium bromide (Tokyo Chemical Industry Co., Ltd.; https://www. tcichemicals.com/eshop/en/jp/commodity/E0370/) for $30 \mathrm{~min}$ and observed by LuminoGraph 2 (ATTO Corporation; https://www.atto.co.jp/eng). Then, this DNA fragment was added to human pooled genomic DNA (Promega Corporation) to prepare several spiked-in samples with different concentrations for a validation of lower limit of detection. In the present study, 1500.0, 150.0, 75.0, 15.0, 7.5, 3.0, and 1.5 copies of DNA fragment harboring EGFR L858R mutation were added to 1500 copies of genomic DNA, resulting in mutation rates of $50.00,9.09,4.76,0.99,0.50,0.20,0.10$ and $0.00 \%$, respectively. Genetic analysis of each of these samples was conducted three times using PCR and LAMP.

Direct sequence method. Direct sequencing of EGFR exon 19, S7681 and G719X regions were conducted by using BigDye ${ }^{\circledR}$ Direct Cycle Sequencing Kit (Thermo Fisher Scientific, 

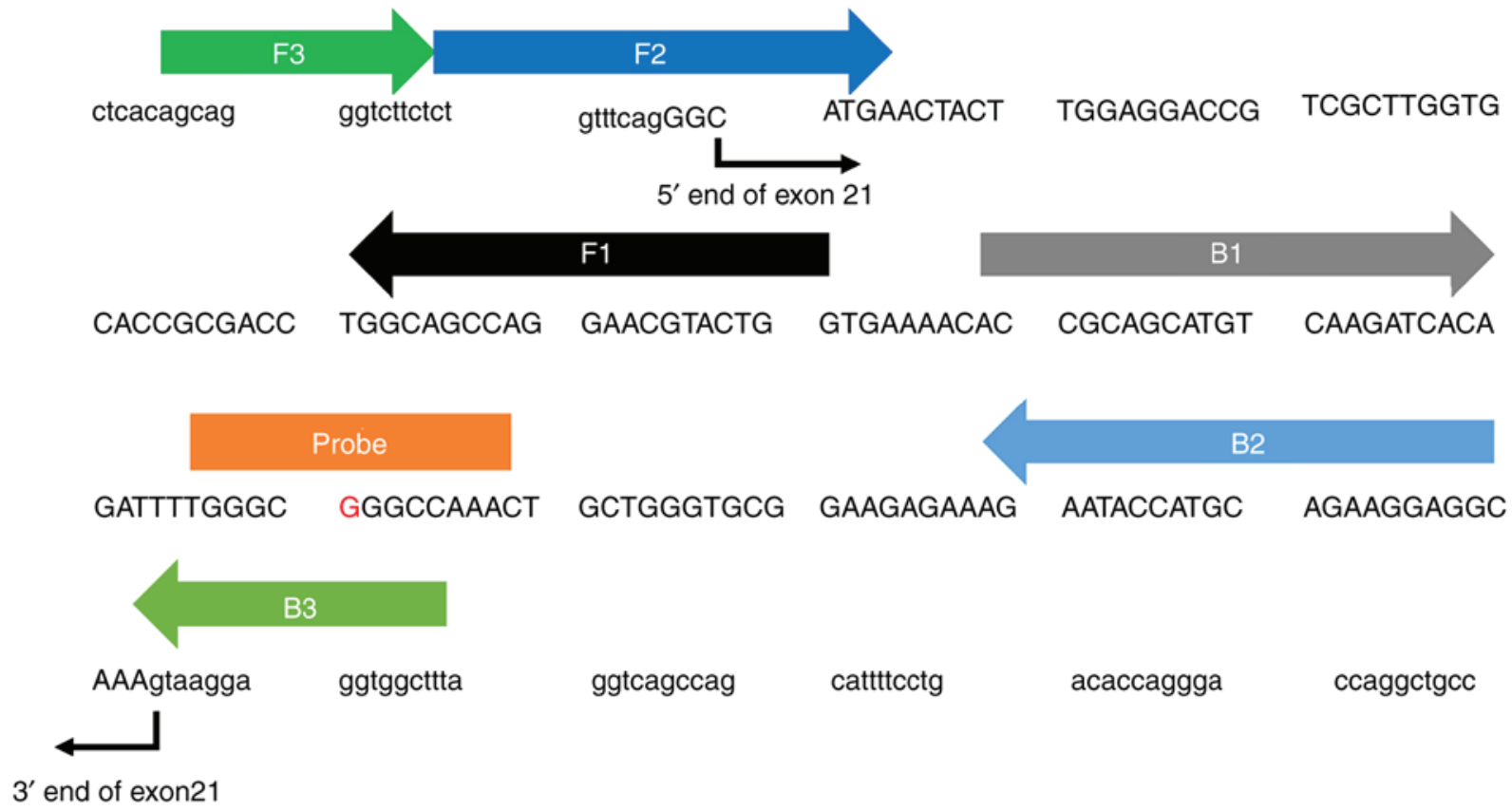

$3^{\prime}$ end of exon21

Figure 1. A schematic view of the association between the EGFR gene and the primers of LAMP assay with forward and backward outer primers (F3 and B3), forward and backward inner primers (FIP and BIP) and forward and back-loop primers (LF and LB). EGFR, epidermal growth factor receptor; LAMP, loop-mediated isothermal amplification.

Inc.) and a ABI PRISM 310 Genetic Analyzer ${ }^{\circledR}$ (Applied Biosystems; Thermo Fisher Scientific, Inc.) to confirm the EGFR mutation status in Case-X. The data were analyzed using Genetyx (version 13.0; https://www.genetyx.co.jp/) and FinchTV (version 1.4.0; https://digitalworldbiology. com/FinchTV). The primers had the following sequences: Exon 19, forward TCCCAGAAGGTGAGAAAGT, reverse: AGAGCTAGAAAGGGAAAGACA; S768I:, forward: CCT CCAGGAAGCCTACGT, reverse: GTCTTTGTGTTCCCG GACAT; and G719X, forward: CCCTTGTCTCTGTGTTCT TG, reverse: TACACCGTGCCGAACGC.

Statistical analysis. All statistical analysis performed using SPSS version 24 for Windows ${ }^{\circledR}$ (IBM Corp). The clinical sensitivity, specificity, positive predictive value (PPV), negative predictive value (NPV) and accuracy of conventional PCR testing of the LAMP method were calculated through comparison with the Therascreen PCR, which was considered as the current gold standard test for detecting EGFR mutations in the present study.

\section{Results}

Characteristics of patients with pulmonary adenocarcinoma. Mean age of the 59 patients was 69.6 years and included 28 males and 31 females. Of these, 27 were non-smokers (45.8\%). The number in pathological stage IA1, IA2, IA3, IB, IIA, IIB, IIIB was 14 (23.7\%), 20 (33.9\%), 6 (10.2\%), 4 (6.8\%), 1 (1.7\%), 3 (5.1\%), and 11 (18.6\%), respectively. All patients had been diagnosed with pulmonary adenocarcinoma, including of 34 papillary predominant (57.6\%), 7 solid predominant (11.9\%), 3 acinar predominant (5.1\%), 7 invasive mucinous $(11.9 \%), 4$ lepidic predominant $(6.8 \%)$ and 4 minimally invasive adenocarcinoma cases (6.8\%; all Table I).
Therascreen EGFR PCR mutation analysis. Among 59 pulmonary tumors, there were 26 tumors with EGFR wild type and 33 tumors with EGFR mutations (Table II). Overall, 18 exon 21 L858R point mutations (54.5\%), 12 exon 19 deletions (36.4\%), 2 simultaneous exon 18 G719X point mutation/exon 20 S768I point mutation (6.1\%) and 1 exon 20 SS761I point mutation $(3.0 \%)$ were detected in the pulmonary tumors with EGFR mutations (Table II).

LAMP EGFR mutation analysis. The LAMP assay detected 27 EGFR wild types and 32 EGFR mutations (Table II). Among 32 EGFR mutations, 18 exon 21 L858R point mutations (54.5\%), 11 exon 19 deletions (33.3\%), 2 simultaneous exon 18 G719X point mutation/exon 20 S768I point mutations (6.1\%) and 1 exon 20 SS761I point mutation alone (3.0\%) were identified (Table II).

Comparison of results of Therascreen PCR and LAMP assays. Table II demonstrates that 33 EGFR mutations in the LAMP assay (18 samples with exon 21 L858R, 11 samples with exon 19 deletions, 1 sample with exon 20 S768I, and 2 samples with exon 18 G719X and exon 20 S768I), and that 32 EGFR mutations in the Therascreen assay (18 samples with exon 21 L858R, 12 samples with exon 19 deletions, 1 sample with exon 20 S768I, and 2 samples with exon 18 G719X and exon 20 S768I); i.e., there was one case showing EGFR wild type of the LAMP but one deletion mutation in exon 19 of the Therascreen PCR (Table III). As assuming that the results of Therascreen PCR assay was true, qualitative analysis was performed for calculating sensitivity, specificity, PPV, NPV and accuracy of the LAMP assay. These values were $97.0 \%$, 100.0, 100.0, 96.3 and 98.3\%, respectively. Furthermore, a genetic analysis was performed to evaluate the detection rate of EGFR mutations in different concentrations. The minimum 
Table I. Clinical characteristics of patients with pulmonary adenocarcinoma.

\begin{tabular}{|c|c|}
\hline Characteristic & $\mathrm{n}(\%)$ \\
\hline Age, years \pm standard deviation & $69.6 \pm 7.1$ \\
\hline$<40$ & $0(0.00)$ \\
\hline $41-50$ & $1(0.02)$ \\
\hline $51-60$ & $5(0.08)$ \\
\hline$>60$ & $53(89.80)$ \\
\hline \multicolumn{2}{|l|}{ Sex } \\
\hline Male & $28(47.5)$ \\
\hline Female & $31(52.5)$ \\
\hline \multicolumn{2}{|l|}{ Smoking status } \\
\hline Never smoker & $27(45.8)$ \\
\hline Current or former smoker & $32(55.2)$ \\
\hline \multicolumn{2}{|l|}{ Tumor location } \\
\hline Right lung & $34(57.6)$ \\
\hline Left lung & $25(42.4)$ \\
\hline \multicolumn{2}{|l|}{ Histology } \\
\hline Papillary predominant & $34(57.6)$ \\
\hline Solid predominant & $7(11.9)$ \\
\hline Acinar predominant & $3(5.1)$ \\
\hline Invasive mucinous predominant & $7(11.9)$ \\
\hline Lepidic predominant & $4(6.8)$ \\
\hline Minimally invasive predominant & $4(6.8)$ \\
\hline \multicolumn{2}{|l|}{ Pathological stage } \\
\hline IA 1 & $14(23.7)$ \\
\hline IA2 & $20(33.9)$ \\
\hline IA3 & $6(10.2)$ \\
\hline IB & $4(6.8)$ \\
\hline IIA & $1(1.7)$ \\
\hline IIB & $3(5.1)$ \\
\hline IIIB & 11 (18.6) \\
\hline
\end{tabular}

concentration for EGFR mutation detection was $4.8 \%$ of DNA sample in Therascreen assay (Table SI). On the other hand, $0.1 \%$ was the minimum concentration in LAMP assay, since LAMP assay demonstrated one success of the detection per 3 tests at the level of $0.1,0.5$ and $1.0 \%$ concentrations and all positive per 3 tests at $>4.8 \%$ concentration (Table SI).

Direct sequencing of Case $X$. It was observed that a single case deviated in the identification of the mutation in exon 19 between Therascreen PCR and the LAMP assay. To confirm this result, direct sequencing of the target site of exon 19 was performed. Direct sequencing results demonstrated no mutation in exon 19 in Case X, which was concordant with the results of the LAMP assay.

To reconfirm the status of EGFR mutation in Case X, four additional FFPE tissue blocks in Case $\mathrm{X}$ were used to extract further DNA samples. The hematoxylin-eosin (HE) images of these FFPE blocks are presented in Fig. 2. Following removal of normal lung tissues, DNA samples were extracted as aforementioned, and investigated using Therascreen EGFR PCR and
Table II. EGFR mutations possessed by patients with pulmonary adenocarcinoma identified using Therascreen and LAMP assays.

\begin{tabular}{lcc}
\hline EGFR mutation & Therascreen & LAMP \\
\hline Exon 21 L858R & 18 & 18 \\
Exon 19 deletions & 12 & 11 \\
Exon 20 S768I & 1 & 1 \\
Exon 18 G719X and exon 20 S768I & 2 & 2 \\
Total & 33 & 32 \\
\hline
\end{tabular}

EGFR, epidermal growth factor receptor; LAMP, loop-mediated isothermal amplification.

Table III. Association between the results between the LAMP assay and the Therascreen assay.

\begin{tabular}{llcc}
\hline & & \multicolumn{2}{c}{ Therascreen PCR } \\
\cline { 3 - 4 } & EGFR status & Positive & Negative \\
\hline \multirow{2}{*}{ LAMP } & Positive & 32 & 0 \\
& Negative & 1 & 26 \\
\hline
\end{tabular}

EGFR, epidermal growth factor receptor.

a LAMP assay. In all the four samples, the deletion mutation in exon 19 was identified using both Therascreen PCR and LAMP assays. Furthermore, direct sequencing revealed a novel exon 19 EGFR deletion mutation in samples a and b; NG_007726.3: g.160744_160761delinsGCA represented the deletion of nucleotides g.160744 to g.160761 (ATTAAGAGAAGCAACATC, data not shown), which were replaced by a GCA nucleotide triplet, changing GGAATTAAGAGAAGCAACATCTCC to GGAGCATCC (data not shown), resulting in shortening substation in the protein (p.Leu747_Ser752delinsHis).

\section{Discussion}

EGFR mutations in pulmonary adenocarcinoma are associated with sensitivity to TKI therapy. Hence the identification of EGFR mutations has become a standard analysis in the treatment pathway of patients with pulmonary adenocarcinoma. Although there are a number of methods available, there is no standardized approach to satisfy the practical clinical requirements of simplicity, rapid and cheap. Several PCR based methods have previously been used as a routine test for the detection of EGFR status in United States, European Union, Japan and China, including the Scorpion Amplification Refractory Mutation System (ARMS) ${ }^{\circledR}(22)$, such as Therascreen PCR assay. This method was approved by the FDA as a standard approach for EGFR gene analysis in lung cancer (fda. gov/medical-devices/recently-approved-devices/therascreenr-fg fr-rgq-pcr-kit-p180043); however, although stable and reliable, this approach has procedural complexities, including complex settings and controls for the temperature at several times using a 


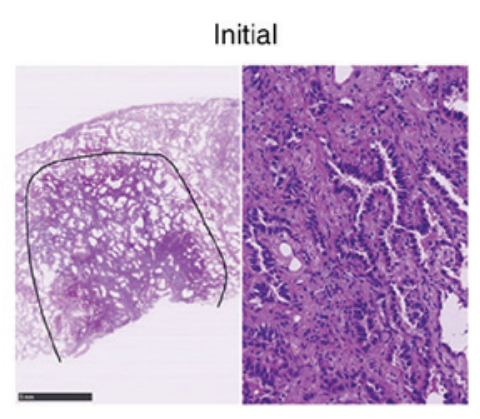

A
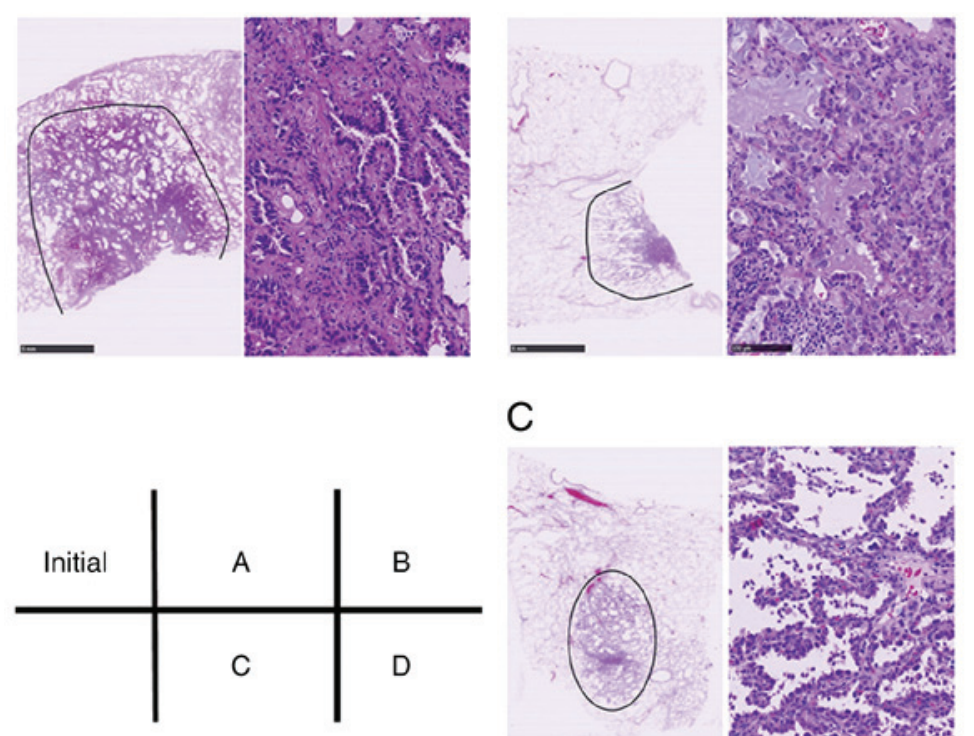

C

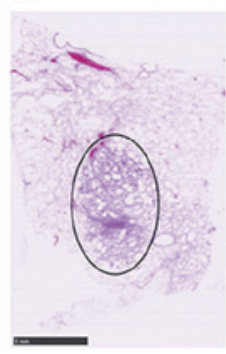

$\mathrm{B}$

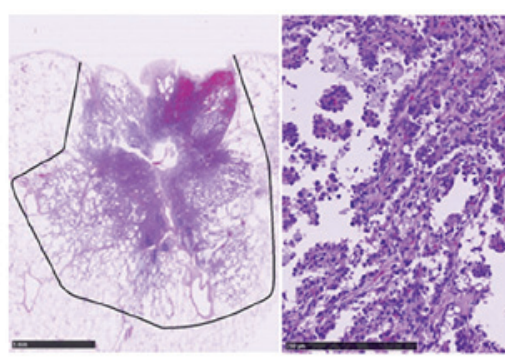

D

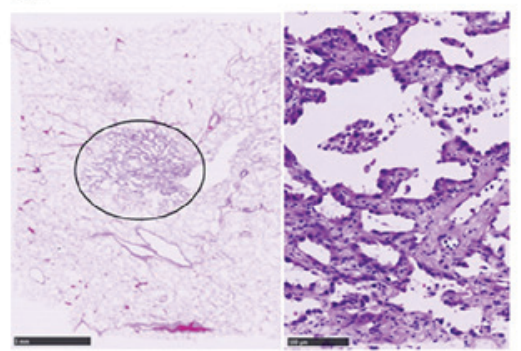

Figure 2. Low and high-power fields of HE-stained formalin-fixed and paraffin-embedded sections of Case X papillary adenocarcinoma. Each the tumor content ratio was $\sim 100 \%$ after trimming along the tumor margin and removing normal lung tissue. The tumor margin is presented as a dotted line. (Initial) LAMP assay reported no EGFR mutation. (A-D) Additional experiments reported an EGFR mutation. (A and B) Direct sequence confirmed a novel EGFR mutation. HE, hematoxylin-eosin; LAMP, loop-mediated isothermal amplification; EGFR, epidermal growth factor receptor.

thermal cycler. Alternatives, such as PCR-Invader ${ }^{\circledR}$ (23), peptide nucleic acid-locked nucleic acid (PNA-LNA) PCR clamp (24) and Cycleave PCR ${ }^{\mathrm{TM}}$ (25), have been developed in Japan and are commercially used in centralized laboratories. The sensitivity of the PNA-LNA PCR clamp is $>97 \%$ with $100 \%$ specificity (26) and the accuracy of Cycleave PCR is $96.7 \%$ (27), so it was the same for our results as it was those.

LAMP is a new PCR method and is considered to be a robust approach for gene analysis as it does not require sophisticated or expensive equipment, such as a thermal cycler necessary for PCR (15). Therefore, the LAMP method may have potential to decrease the costs of gene analysis. Previous studies have demonstrated the value of LAMP in field of bacteriology and virology $(15,17,28,29)$; however, few studies have reported the value of LAMP in oncology. Ikeda et al (30) used LAMP assays to detect EGFR mutations in NSCLC, demonstrating the value of LAMP, but only the L858R mutation was studied. Therefore, the present study aimed to investigate other EGFR mutations, including those in exon 19, exon 21 and other minor mutations using LAMP assays.

The sensitivity, specificity, PPV, NPV and accuracy values of LAMP for EGFR mutations compared with the Therascreen assay method were 97, 100,100,96.3 and 98.3\%, respectively, demonstrating the potential of LAMP as an efficient alternative approach $t$ oncogene mutation analyses. To evaluate the sensitivity of these two assays, a genetic analysis was performed to investigate the minimum concentration of EGFR mutation detection in both methods. The minimum concentration for LAMP was $4.8 \%$ and there were no detection under $1.0 \%$ in the Therascreen assay. Therefore, the minimum concentration for the LAMP assay is $\sim 50$ times higher than $4.8 \%$ in the Therascreen assay. With almost equal efficiency, detection of the exon 19 mutation in Case X using the Therascreen method suggested that LAMP, as well as direct sequence methods, could identify false negatives. However, additional experiments investigating Case $\mathrm{X}$ tumor tissues demonstrated the presence of deletion mutations in exon 19. The direct sequence method has reported low sensitivity $(31,32)$, whereas the Therascreen method was generally recognized as a promising method $(11,12)$. However, the identified mutation should be simplified, and an improved, faster and cheaper method of identification should be developed for its clinical application.

The additional Case $\mathrm{X}$ experiments also identified a novel EGFR mutation using direct sequencing which was not identified using Therascreen or LAMP; however, this mutation may have been detected as an exon 19 deletion by the primers of the similarly targeted mutation. However, the details of the primer of LAMP method could not be disclosed due to the policies of Eiken Co., Ltd., and further information concerning the primers of Therascreen EGFR PCR kit could not be obtained due to the patent. Furthermore, the present study noted that this novel mutation was not included in the COSMIC database and that no previous studies had reported this mutation. Therefore, the present study is the first report this EGFR mutation, to the best of our knowledge.

In conclusion, the LAMP method may be a valuable alternative for the identification of oncogenic mutations in lung cancer. Currently, the study group is developing a new method of detecting oncogenes using liquid biopsies (data not shown). In addition, a novel mutation, NG_007726.3:g.160744_16076 1delinsGCA, was identified exon 19 of EGFR. However, this needs further validation before clinical use.

\section{Acknowledgements}

The authors would like to thank Mr Satoru Michiyuki, employee of Eiken Chemical Co., Ltd., Otawara, Japan, for his valuable comments and suggestions concerning the LAMP 
assay; and Mr. Yasuhito Kobayashi, Mr. Hidehiro Numagami and Ms. Mei Miyagawa of the Saitama Cardiovascular and Respiratory Center, Kumagaya, Japan, for their technical assistance.

\section{Funding}

The present study was funded by Eiken Chemical Co., Ltd.

\section{Availability of data and materials}

The datasets used and/or analyzed during the present study are available from the corresponding author upon reasonable request.

\section{Authors' contributions}

SH analyzed the data and wrote the initial draft of the manuscript. YS designed the study, analyzed and interpreted the data and assisted in preparing the manuscript. AM performed all experiments and analyzed all results in the study. YS made pathological diagnosis in all cases and provided all tissue samples. NT, TI and EH contributed to data collection and interpretation. MY performed some of the experiments, and critically reviewed the manuscript, and organized research group in this study. Besides AM, all authors approved the final version of the manuscript and they agree to be accountable for all aspects of the work.

\section{Ethical approval and consent to participate}

The present study was approved by The Institutional Review Board of the Saitama Cardiovascular and Respiratory Center (approval no. 2016015). Written informed consent was provided by all patients.

\section{Patient consent for publication}

Not applicable.

\section{Competing interests}

AM is an employee of Eiken Chemical Co., Ltd, and was the only author who conducted all the experiments. Eiken Chemical Co., Ltd, provided the research grant for the present study, and provided the LAMP assay which is not currently commercially available. Eiken Chemical Co., Ltd., had no control over the interpretation, writing, or publication of the present study.

\section{References}

1. Jemal A, Bray F, Center MM, Ferlay J, Ward E and Forman D: Global cancer statistics. CA Cancer J Clin 61: 69-90, 2011.

2. Siegel R, Naishadham D and Jemal A: Cancer statics, 2013. CA Cancer J Clin 63: 11-30, 2013.

3. Travis WD, Brambilla E, Burke AP, Marx A and Nicholson AG Introduction to the 2015 world health organization classification of tumors of the lung, pleura, thymus, and heart. J Thorac Oncol 10: 1240-1242, 2015

4. Lin JJ, Cardarella S, Lydon CA, Dahlberg SE, Jackman DM, Jänne PA and Johnson BE: Five-year survival in EGFR-mutant metastatic lung adenocarcinoma treated with EGFR-TKIs. J Thorac Oncol 11: 556-565, 2016.
5. Lynch TJ, Bell DW, Sordella R, Gurubhagavatula S, Okimoto RA, Brannigan BW, Harris PL, Haserlat SM, Supko JG, Haluska FG, et al: Activating mutations in the epidermal growth factor receptor underlying responsiveness of non-small-cell lung cancer to gefitinib. N Engl J Med 350: 2129-2139, 2004.

6. Kohno T, Tsuta K, Tsuchihara K, Nakaoku T, Yoh K and Goto K: RET fusion gene: Translation to personalized lung cancer therapy. Cancer Sci 104: 1396-1400, 2013.

7. Pao W and Hutchinson KE: Chipping away at the lung cancer genome. Nat Med 18: 349-351, 2012.

8. Li T, Kung HJ, Mack PC and Gandara DR: Genotyping and genomic profiling of non-small-cell lung cancer: Implications for current and future therapies. J Clin Oncol 31: 1039-1049, 2013.

9. Paez JG, Janne PA, Lee JC, Tracy S, Greulich H, Gabriel S, Herman P, Kaye FJ, Lindeman N, Boggon TJ, et al: EGFR mutations in lung cancer: Correlation with clinical response to gefitinib therapy. Science 304: 1497-1500, 2004.

10. De Pas T, Toffalorio F, Manzotti M, Fumagalli C, Spitaleri G, Catania C, Delmonte A, Giovannini M, Spaggiari L, de Braud F and Barberis M: Activity of epidermal growth factor receptor-tyrosine kinase inhibitors in patients with non-small cell lung cancer harboring rare epidermal growth factor receptor mutations. J Thorac Oncol 6: 1895-1901, 2011.

11. Wu YL, Zhou C, Liam CK, Wu G, Liu X, Zhong Z, Lu S, Cheng Y, Han B, Chen L, et al: First-line erlotinib versus gemcitabine/cisplatin in patients with advanced EGFR mutation-positive non-small-cell lung cancer: Analyses from the phase III, randomized, open-label, ENSURE study. Ann Oncol 26: 1883-1889, 2015.

12. Yang JC, Wu YL, Schuler M, Sebastian M, Popat S, Yamamoto N, Zhou C, Hu CP, O'Byrne K, Feng J, et al: Afatinib versus cisplatin-based chemotherapy for EGFR mutation-positive lung adenocarcinoma (LUX-Lung 3 and LUX-Lung 6): Analysis of overall survival data from two randomised, phase 3 trials. Lancet Oncol 16: 141-151, 2015.

13. Garinet S, Laurent-Puig P, Blons H and Oudart JB: Current and future molecular testing in NSCLC, what can we expect from new sequencing technologies? J Clin Med 7: E144, 2018.

14. Marino P, Touzani R, Perrier L, Rouleau E, Kossi DS, Zhaomin Z, Charrier N, Goardon N, Preudhomme C, Durand-Zaleski I, et al: Cost of cancer diagnosis using next-generation sequencing targeted gene panels in routine practice: A nationwide French study. Eur J Hum Genet 26: 314-323, 2018.

15. Notomi T, Okayama H, Masubuchi H, Yonekawa T, Watanabe K, Amino $\mathrm{N}$ and Hase T: Loop-mediated isothermal amplification of DNA. Nucleic Acids Res 28: E63, 2000.

16. Yoshino M, Annaka T, Kojima T and Ikedo M: Sensitive and rapid detection of mycoplasma pneumoniae by loop-mediated isothermal amplification. Kansenshogaku Zasshi 82: 168-176, 2008 (In Japanese)

17. Poon LL, Leung CS, Tashiro M, Chan KH, Wong BW, Yuen KY, Guan Y and Peiris JS: Rapid detection of the severe acute respiratory syndrome (SARS) coronavirus by a loop-mediated isothermal amplification assay. Clin Chem 50: 1050-1052, 2004

18. Imai M, Ninomiya A, Minekawa $H$, Notomi T, Ishizaki T, Van Tu P, Tien NT, Tashiro M and Odagiri T: Rapid diagnosis of H5N1 avian influenza virus infection by newly developed influenza H5 hemagglutinin gene-specific loop-mediated isothermal amplification method. J Virol Methods 141: 173-180, 2007.

19. Goldstraw P, Chansky K, Crowley J, Rami-Porta R, Asamura H, Eberhardt WE, Nicholson AG, Groome P, Mitchell A, Bolejack V, et al: The IASLC lung cancer staging project: Proposals for revision of the TNM stage groupings in the forthcoming (eighth) edition of the TNM Classification for lung cancer. J Thorac Oncol 11: 39-51, 2016.

20. Angulo B, García-García E, Martínez R, Suárez-Gauthier A, Conde E, Hidalgo M and López-Ríos F: A commercial real-time PCR kit provides greater sensitivity than direct sequencing to detect KRAS mutations: A morphology-based approach in colorectal carcinoma. J Mol Diagn 12: 292-299, 2010.

21. Vallée A, Le Loupp AG and Denis MG: Efficiency of the Therascreen ${ }^{\circledR}$ RGQ PCR kit for the detection of EGFR mutations in non-small cell lung carcinomas. Clin Chim Acta 429: 8-11, 2014.

22. Kimura H, Kasahara K, Kawaishi M, Kunitoh H, Tamura T, Holloway B and Nishio K: Detection of epidermal growth factor receptor mutations in serum as a predictor of the response to gefitinib in patients with non-small-cell lung cancer. Clin Cancer Res 12: 3915-3921, 2006. 
23. Hall JG, Eis PS, Law SM, Reynaldo LP, Prudent JR, Marshall DJ, Allawi HT, Mast AL, Dahlberg JE, Kwiatkowski RW, et al: Sensitive detection of DNA polymorphisms by the serial invasive signal amplification reaction. Proc Natl Acad Sci USA 97: 8272-8277, 2002.

24. Nagai Y, Miyazawa H, Huqun, Tanaka T, Udagawa K, Kato M, Fukuyama S, Yokote A, Kobayashi K, Kanazawa M and Hagiwara K: Genetic heterogeneity of the epidermal growth factor receptor in non-small cell lung cancer cell lines revealed by a rapid and sensitive detection system, the peptide nucleic acid-locked nucleic acid PCR clamp. Cancer Res 65: 7276-7282, 2005.

25. Yatabe $Y$, Hida T, Horio $Y$, Kosaka T, Takahashi $T$ and Mitsudomi T: A rapid, sensitive assay to detect EGFR mutation in small biopsy specimens from lung cancer. J Mol Diagn 8: $335-341,2006$

26. Tanaka T, Nagai Y, Miyazawa H, Koyama N, Matsuoka S, Sutani A, Huqun, Udagawa K, Murayama Y, Nagata M, et al: Reliability of the peptide nucleic acid-locked nucleic acid polymerase chain reaction clamp-based test for epidermal growth factor receptor mutations integrated into the clinical practice for non-small cell lung cancers. Cancer Sci 98: 246-252, 2007.

27. Nakamura H, Koizumi H, Sakai H, Kimura H, Miyazawa T, Marushima H, Saji H and Takagi M: Accuracy of the cobas EGFR mutation assay in non-small-cell lung cancer compared with three laboratory-developed tests. Clin Lung Cancer 19: 170-174, 2018.

28. Waites KB, Xiao L, Liu Y, Balish MF and Atlinson TP: Mycoplasma pneumoniae from the respiratory tract and beyond. Clin Microbiol Rev 30: 747-809, 2017.
29. Dinh DT, Le MT, Vuong CD, Hasebe F and Morita K: An updated loop-mediated isothermal amplification method for rapid diagnosis of $\mathrm{H} 5 \mathrm{~N} 1$ avian influenza viruses. Trop Med Health 39: 3-7, 2011

30. Ikeda S, Takabe K, Inagaki M, Funakoshi $\mathrm{N}$ and Suzuki K: Detection of gene point mutation in paraffin sections using in situ loop-mediated isothermal amplification. Pathol Int 57: 594-599, 2007.

31. Fan X, Furnari FB, Cavenee WK and Castresana JS: Non-isotopic silver-stained SSCP is more sensitive than automated direct sequencing for the detection of PTEN mutations in a mixture of DNA extracted from normal and tumor cells. Int J Oncol 18: 1023-1026, 2001.

32. Endo K, Konishi A, Sasaki H, Takada M, Tanaka H, Okumura M, Kawahara M, Sugiura H, Kuwabara Y, Fukai I, et al: Epidermal growth factor receptor gene mutation in non-small cell lung cancer using highly sensitive and fast TaqMan PCR assay. Lung Cancer 50: 375-384, 2005.

This work is licensed under a Creative Commons Attribution-NonCommercial-NoDerivatives 4.0 International (CC BY-NC-ND 4.0) License. 